\title{
SHIFT OF BIFURCATION POINT DUE TO NOISE INDUCED PARAMETER
}

\author{
SANDIP BANERJEE, RAKHI BHATTACHARYA, and C. G. CHAKRABARTI
}

(Received 15 June 1998)

\begin{abstract}
The object of the paper is to see the effect of small stochastic parametric perturbation on a nonlinear interacting system exhibiting Hopf bifurcation. The method is based on the technique of Markov diffusion approximation.
\end{abstract}

Keywords and phrases. Stochastic perturbation, Hopf bifurcation, spectral density, limit cycle, Markov process.

2000 Mathematics Subject Classification. Primary 37G15.

1. Introduction. The study of nonlinear stochastic dynamical systems has received a great deal of attention in the past three decades and nonlinear systems, which are extremely excited have been explored in most of these studies [4]. Lin [6] has carried out the analysis by the Markov diffusion approximation in the case of wide-band case excitation. Ariaratanam [1, 2] studied the behavior of linear conservative stochastic systems under parametric excitation.

In this paper, the influence of small stochastic perturbation on a nonlinear, nonconservative interacting population exhibiting Hopf bifurcation has been investigated. It is observed that due to the presence of parametric perturbation, the bifurcation point is shifted.

2. Basic stochastic differential equations. Let us consider an interacting population of two species whose concentrations are denoted by $a(t)$ and $b(t)$ governed by the system of equation [7]

$$
\begin{aligned}
& \dot{a}=\gamma a-\omega b+(m a-n b)\left(a^{2}+b^{2}\right), \\
& \dot{b}=\omega a+\gamma b+(n a+m b)\left(a^{2}+b^{2}\right),
\end{aligned}
$$

where $\gamma$ is a scalar control parameter and $m, n, \omega$ are constants. The equilibrium point of the system is $(0,0)$. The given system is expressed as

$$
\frac{d \Theta(t)}{d t}=\Omega(\Theta(t), \gamma), \quad \text { where } \Theta(t)=\left[\begin{array}{l}
a(t) \\
b(t)
\end{array}\right],
$$

and

$$
\Omega(\Theta, \gamma)=\left[\begin{array}{l}
\gamma a-\omega b+(m a-n b)\left(a^{2}+b^{2}\right) \\
\omega a+\gamma b+(n a+m b)\left(a^{2}+b^{2}\right)
\end{array}\right] .
$$


Considering the random environment, we extend this system to the stochastic differential equation

$$
\frac{d \Theta(t)}{d t}=\Omega(\Theta(t), \gamma)+\varepsilon \lambda(t) \Theta(t),
$$

where

$$
\lambda(t)=\left[\begin{array}{cc}
k(t) & -p(t) \\
k(t) & 0
\end{array}\right]
$$

where $k(t)$ and $p(t)$ are uncorrelated stationary stochastic process with zero mean and $\varepsilon$ is a small parameter, $|\varepsilon| \ll 1$. Here, $k(t)$ and $p(t)$ are considered as processes with arbitrary smoothly varying spectral density function with small correlation time.

The Jacobian matrix $J(\gamma)$ of $\Omega$ at $(0,0)$ has two complex eigenvalues, $\gamma \pm i \omega$. As $\gamma$ passes through $\gamma=0$, the real part of the eigenvalues changes from negative (stable focus) to positive (unstable focus). Therefore, $\gamma=0$ is a bifurcating point. When $\gamma=0$, the eigenvalues of $J(\gamma)$ are purely imaginary. These values are $\lambda_{1}, \lambda_{2}= \pm i \omega$.

The linear problem corresponding to equation (2.1) has direct and adjoint eigenvalue problems

$$
J(0) x_{1}=\lambda_{1} x_{1}
$$

where $x_{1}=\alpha_{1}+i \beta_{1}=\left[\begin{array}{l}1 \\ 0\end{array}\right]+i\left[\begin{array}{l}0 \\ 1\end{array}\right]$,

$$
J^{T}(0) x_{2}=\lambda_{2} x_{2},
$$

where $x_{2}=\alpha_{2}+i \beta_{2}=\left[\begin{array}{l}1 \\ 0\end{array}\right]+i\left[\begin{array}{c}0 \\ -1\end{array}\right]$, respectively.

We consider the transformation [3]

$$
\Theta(t)=2 \varepsilon x_{1}\left(\alpha_{1} \cos \Phi+\beta_{1} \sin \Phi\right), \quad \text { where } \Phi=\omega t+\Psi,
$$

where $x_{1}$ and $\Psi$ are the amplitude and the phase of the solution.

Multiplying (2.4) by $x_{2}{ }^{T} \dot{\Theta}$ and considering the transformation (2.8), (2.4) can be reduced to the following equations (neglecting the terms of order higher than $\varepsilon^{2}$ ):

$$
\begin{aligned}
& \dot{x}_{1}=\varepsilon\left\{x_{1}\left[\frac{(1+\cos 2 \Phi+\sin 2 \Phi)}{2} k(t)-\frac{1}{2} \sin 2 \Phi p(t)\right]-\varepsilon\left(x_{1} v+4 x_{1}^{3} m\right)\right\}, \\
& \dot{\Psi}=\varepsilon\left\{\left[\frac{(1+\cos 2 \Phi-\sin 2 \Phi)}{2} k(t)+\frac{(1-\cos 2 \Phi)}{2} p(t)\right]+\left(4 x_{1}^{2} \varepsilon n\right)\right\},
\end{aligned}
$$

where $\gamma=\varepsilon^{2} v$.

Now, for the system (2.1), we see that $\gamma=0$ is a bifurcation point and at this bifurcating value. We have the corresponding eigenvalues to be $\pm i \omega$, i.e., purely imaginary and

$$
\frac{d(\operatorname{Re} \lambda)}{d \lambda}=1 \neq 0 .
$$

Then by the Hopf bifurcation theorem, we predict the existence of a periodic orbit for the considered equation. 
Now, we want to analyze the stability of the limit cycle in Hopf bifurcation. For this, we have to calculate $V^{\prime \prime \prime}$ at the steady state when $\gamma=0$, where

$$
\begin{aligned}
V^{\prime \prime \prime}(a, b)= & \frac{3 \pi}{4 \omega}\left[f_{a a a}+f_{a b b}+g_{a a b}+g_{b b b}\right] \\
& +\frac{3 \pi}{4 w^{2}}\left[f_{a b}\left(f_{a a}+f_{a b}\right)+g_{a b}\left(g_{a a}+g_{b b}\right)+f_{a a} g_{a a}-f_{b b} g_{b b}\right],
\end{aligned}
$$

where

$$
\begin{aligned}
& f(a, b)=\gamma a-\omega b+(m a-n b)\left(a^{2}+b^{2}\right), \\
& g(a, b)=\omega a+\gamma b+(n a+m b)\left(a^{2}+b^{2}\right) .
\end{aligned}
$$

Therefore,

$$
V^{\prime \prime \prime}(0,0) /_{\gamma=0}=\frac{12 \pi}{\omega} m .
$$

Thus, $V^{\prime \prime \prime}<0$, if $m<0$, then the limit cycle occurs for $\gamma>0$ (supercritical) and is stable and $V^{\prime \prime \prime}>0$, if $m>0$, then the limit cycle occurs for $\gamma<0$ (subcritical) and is repelling, i.e., unstable. However, when $m=0$, although the conditions for a Hopf bifurcation are satisfied, there are no periodic orbits in the vicinity of the bifurcating point.

3. Approximation to Markov process. We assume that $k(t)$ and $p(t)$ are smoothly varying spectral density functions with small correlation time. By applying limit theorem of Stratonovich [9] and Khasminsky [5], we can say that the process converges weakly as $\varepsilon \rightarrow 0$ to a diffusive vector Markov process governed by a pair of Ito equation obtained by applying the stochastic averaging process to equations (2.9) and (2.10). Equations (2.9) and (2.10) are approximated by the Ito equations

$$
\begin{gathered}
d x_{1}=\varepsilon^{2} m_{x_{1}} d t+\varepsilon\left[\sigma_{11} d w_{1}+\sigma_{12} d w_{2}\right], \\
d \Psi=\varepsilon^{2} m_{\Psi} d t+\varepsilon\left[\sigma_{12} d w_{1}+\sigma_{22} d w_{2}\right],
\end{gathered}
$$

where $w_{1}(t)$ and $w_{2}(t)$ are independent Wiener process with unit density and

$$
\begin{gathered}
m_{x_{1}}=\frac{1}{8} \xi_{1}(0)+\frac{3}{8} \xi_{1}(2 \omega)+\frac{3}{8} \xi_{2}(2 \omega)+v, \\
m_{\Psi}=-\frac{1}{4} \Psi_{1}(2 \omega)-\frac{1}{8} \Psi_{2}(2 \omega), \\
\sigma_{11}^{2}+\sigma_{12}^{2}=\frac{1}{8}\left[2 \xi_{1}(0)+2 \xi_{1}(2 \omega)+\xi_{2}(2 \omega)\right], \\
\sigma_{21}^{2}+\sigma_{22}^{2}=\frac{1}{8}\left[2 \xi_{1}(2 \omega)+\xi_{2}(2 \omega)\right], \\
\xi_{i}(\omega)=\int_{0}^{\infty}\left\langle h_{i}(t) h_{i}(t+\tau)\right\rangle \cos \omega \tau d \tau \quad(i=1,2) \\
\Psi_{i}(\omega)=\int_{0}^{\infty}\left\langle h_{i}(t) h_{i}(t+\tau)\right\rangle \sin \omega \tau d \tau,
\end{gathered}
$$


where

$$
h_{1}(t)=k(t) ; \quad h_{2}(t)=p(t) .
$$

The differential equations for the moments of $x_{1}$ are given by

$$
\begin{gathered}
\frac{d\left\langle x_{1}\right\rangle}{d t}=\xi^{2} m_{x_{1}}\left\langle x_{1}\right\rangle, \quad \frac{d\left\langle x_{1}^{2}\right\rangle}{d t}=2 \xi^{2}\left(m_{x_{1}}+L\right)\left\langle x_{1}^{2}\right\rangle, \\
L=\frac{1}{16}\left[2 \xi_{1}(0)+2 \xi_{1}(2 \omega)+\xi_{2}(2 \omega)\right] .
\end{gathered}
$$

Therefore,

$$
D(t)=\left\langle\left(x_{1}-\left\langle x_{1}\right\rangle^{2}\right)\right\rangle=\left\langle x_{1}^{2}\right\rangle-\left\langle x_{1}\right\rangle^{2} .
$$

Solving (3.6) and substituting the values in (3.8), we get,

$$
D(t)=\exp \left[2 \varepsilon^{2}\left(m_{x_{1}}+L\right) t\right]\left\{D_{2}-D_{1} e^{-2 \varepsilon^{2} L t}\right\},
$$

where

$$
\left\{\left\langle x_{1}\right\rangle_{t=0}\right\}^{2}=D_{1}, \quad\left\langle x_{1}^{2}\right\rangle_{t=0}=D_{2} .
$$

For the stability of the system from (3.9), we must have

$$
m_{x_{1}}+L \leq 0
$$

or

$$
v \geq-\frac{1}{4}\left[2 \xi_{1}(0)+2 \xi_{1}(2 \omega)+\frac{1}{4} \xi_{2}(2 \omega)\right] .
$$

Therefore, the system passes from unstable state to stable state as $v$ passes through

$$
v^{*}=-\frac{1}{4}\left[2 \xi_{1}(0)+2 \xi_{1}(2 \omega)+\frac{1}{4} \xi_{2}(2 \omega)\right] .
$$

Thus, there is a shift of bifurcation point due to the noise induced parameter.

Now, let us find the condition of stability of the limit cycle after considering the small stochastic parametric perturbation for which we have to calculate $V^{\prime \prime \prime}$ at steady state and $\gamma=\gamma^{*}$, where

$$
\gamma^{*}=-\frac{\varepsilon^{2}}{4}\left[\xi_{1}(0)+2 \xi_{1}(2 \omega)+\frac{7}{4} \xi_{2}(2 \omega)\right] .
$$

From (2.14), we see that $V^{\prime \prime \prime}$ is independent of $\gamma$ and depends on $m$. The limit cycle occurs and is unstable or stable according to whether

$$
\gamma \lessgtr-\frac{\varepsilon^{2}}{4}\left[\xi_{1}(0)+2 \xi_{1}(2 \omega)+\frac{7}{4} \xi_{2}(2 \omega)\right] .
$$


4. Conclusion. The bifurcation theory plays a significant role in the behavior of the linear system. Although it is self-developed for deterministic system, it is still in infancy for stochastically perturbed or excited systems [8]. In this paper, the interacting population governed by nonlinear system of equations has a Hopf bifurcation, which generates an unstable or stable limit cycle according as whether the bifurcation parameter is negative or positive. The stochastic analysis of the Hopf bifurcation of nonlinear interacting population system under the influence of small stochastic parametric excitation due to the fluctuating environment has been studied. We see that, due to this noise-induced parameter, the bifurcation point is shifted but the limit cycle, which occurs still remains unstable or stable according to whether $\gamma$ less than or greater than $\gamma^{*}$. The system of the nonlinear equations considered, though has no direct biological significance, it could still be considered as a model of a system of equations for the stochastic Hopf bifurcation analysis of stochastically excited systems.

ACKNOWLEDGEMENT. The first author (SB) wishes to thank the Council of Scientific and Industrial Research (India) for a Senior Research Fellowship.

\section{REFERENCES}

[1] S. T. Ariaratnam and N. Sri-Namachchivaya, Dynamic stability of pipes conveying fluid with stochastic flow velocity, Random Vibration-Status and Recent Developments (Amsterdam, New York) (I. Elishhakoff and R. H. Lyon, eds.), Stud. Appl. Mech., vol. 14, Elsevier, 1986, pp. 1-17. MR 90j:73047.

[2] S. T. Ariaratnam and T. K. Srikantaiah, Parametric instabilities in elastic structures under stochastic loading, J. Structural Mech. 6 (1978), 349-365.

[3] A. Bruckner and Y. K. Lin, Application of complex stochastic averaging to nonlinear random vibration problems, Internat. J. Non-Linear Mech. 22 (1987), no. 3, 237-250. MR 88k:70011. Zbl 612.73094.

[4] W. Horsthemke and R. Lefever, Noise-Induced Transitions, Springer Series in Synergetics, vol. 15, Springer-Verlag, Berlin, New York, 1984, Theory and applications in physics, chemistry, and biology. MR 86e:82001. Zbl 529.60085.

[5] R. Z. Khas'minski, A limit theorem for solutions of differential equations with a random right hand part, Teor. Veroyatnost. i Primenen. 11 (1966), 444-462 (Russian). MR 34\#3637. Zbl 202.48601.

[6] Y. K. Lin, Probabilistic Theory of Structural Dynamics, McGraw-Hill, New York, 1967.

[7] A. H. Nayfeh and B. Balachandran, Applied Nonlinear Dynamics, Wiley Series in Nonlinear Science, John Wiley \& Sons, Inc., New York, 1995, Analytical, computational, and experimental methods. MR 96a:34053. Zbl 848.34001.

[8] N. Sri-Namachchivaya and S. T. Ariaratnam, Stochastically perturbed Hopf bifurcation, Internat. J. Non-Linear Mech. 22 (1987), no. 5, 363-372. MR 89k:70050. Zbl 635.34037.

[9] R. L. Stratonovich, Topics in the Theory of Random Noise. Vol. I: General Theory of Random Processes. Nonlinear Transformations of Signals and Noise, Gordon and Breach Science Publishers, New York, London, 1963. MR 28\#1660. Zbl 183.22007.

BANeRJEe: Department of Applied Mathematics, University of Calcutta, 92, A.P.C. ROAD, CALCUTTA-700009, INDIA

E-mail address: sandip_banerjee@hotmai 1.com

BHATTACHARYA AND CHAKRABARTI: DEPARTMENT OF APPLIED MATHEMATICS, UNIVERSITY OF CALCUTTA, 92, A.P.C. ROAD, CALCUTTA-700009, INDIA 


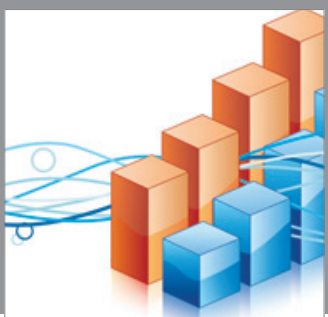

Advances in

Operations Research

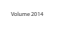

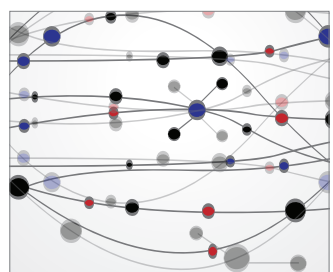

\section{The Scientific} World Journal
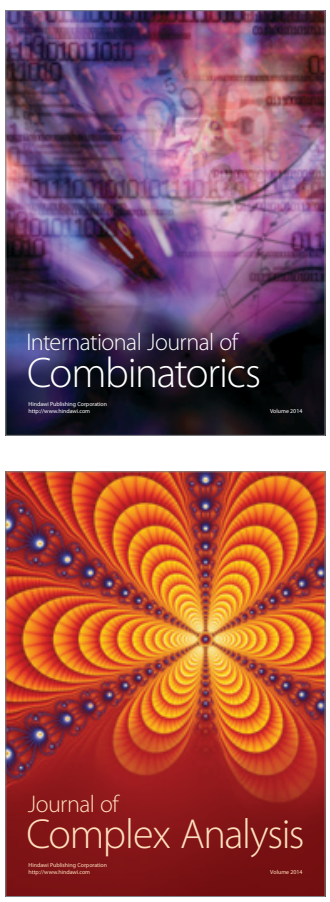

International Journal of

Mathematics and

Mathematical

Sciences
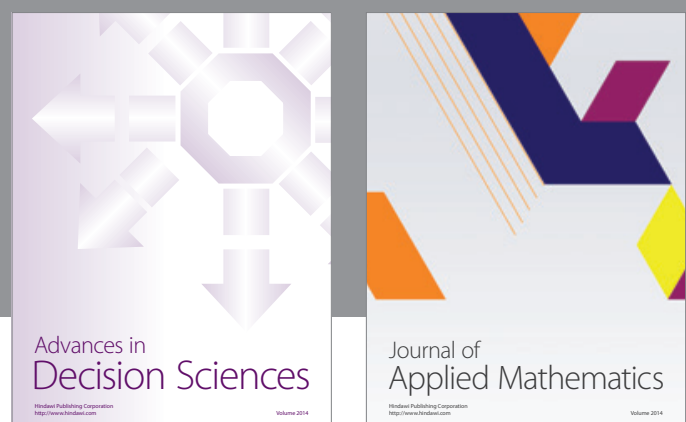

Journal of

Applied Mathematics
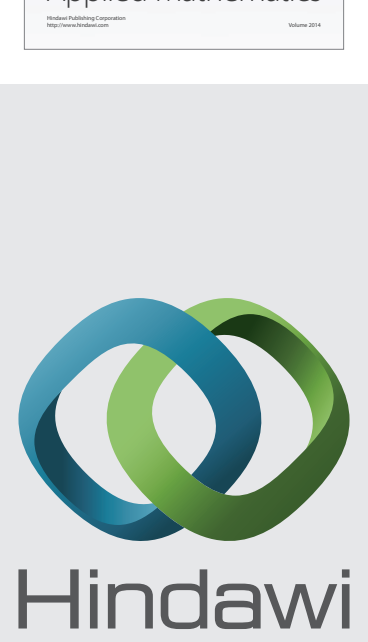

Submit your manuscripts at http://www.hindawi.com
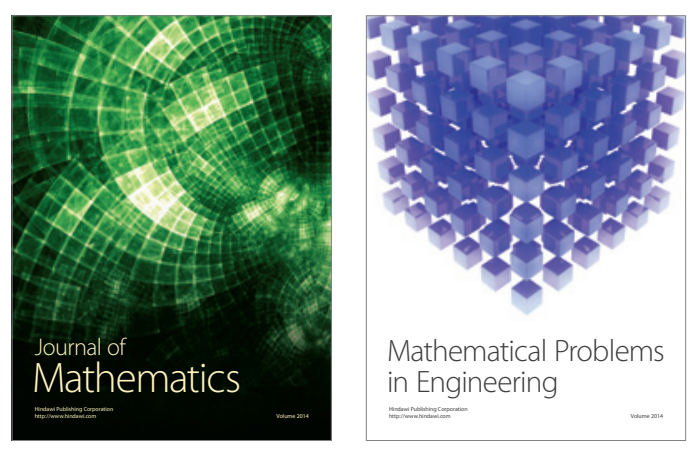

Mathematical Problems in Engineering
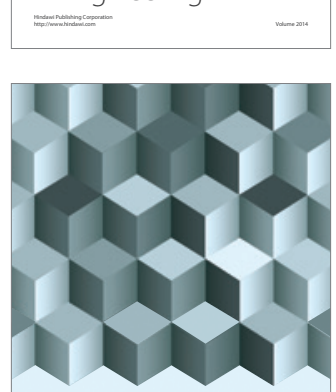

Journal of

Function Spaces
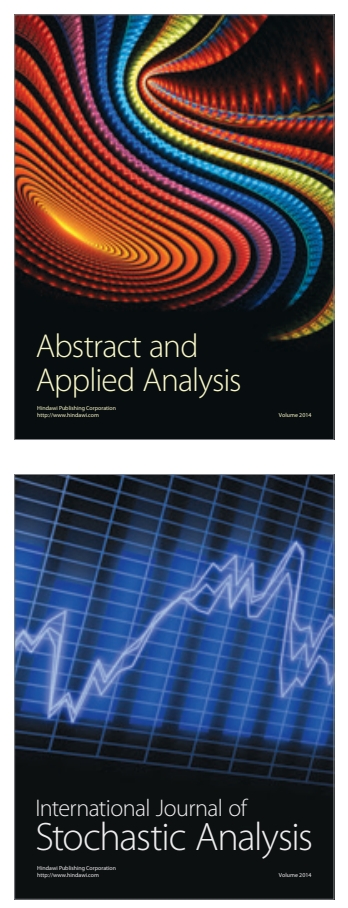

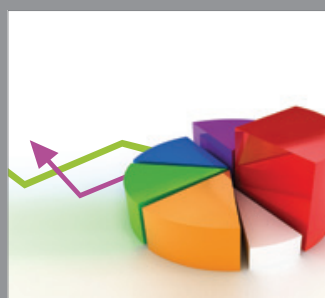

ournal of

Probability and Statistics

Promensencen
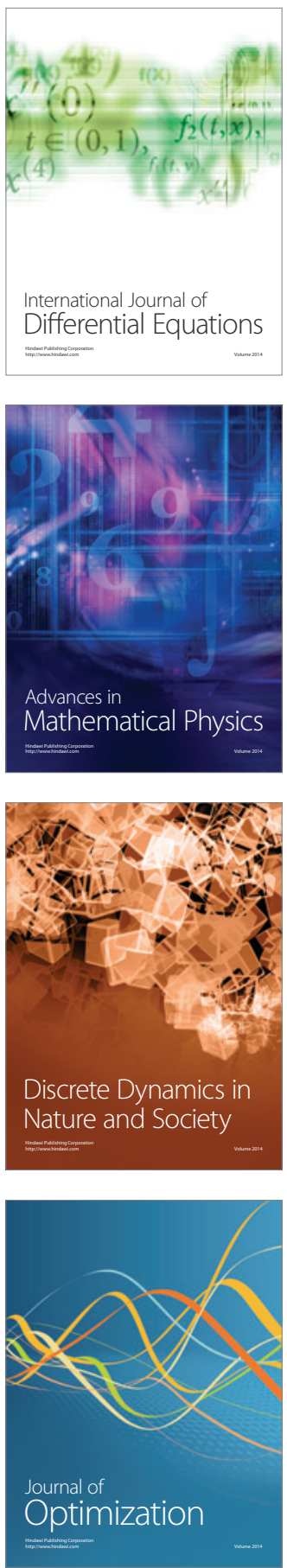